\title{
SEXUAL MOTIVATION IS DEMASCULINIZED, BUT NOT FEMINIZED, IN PRENATALLY STRESSED MALE RATS
}

\author{
C.-T. WANG, ${ }^{a 1}$ H.-A. SHUI, ${ }^{b 1}$ R.-L. HUANG, ${ }^{a}$ M.-Y. TAI, ${ }^{a}$ \\ M.-T. PENG ${ }^{a}$ AND Y.-F. TSAl ${ }^{a *}$ \\ ${ }^{a}$ Department of Physiology, College of Medicine, National Taiwan \\ University, 1 Jen-Ai Road, 1st Section, Taipei, Taiwan (100), Republic \\ of China \\ ${ }^{b}$ Graduate Institute of Medical Sciences, National Defense Medical \\ Center, 161 Min-Chuan East Road, 6th Section, Taipei, Taiwan (114), \\ Republic of China
}

\begin{abstract}
Sexual motivation and copulation in male rats are associated with dopamine release in the nucleus accumbens. Demasculinized copulatory behavior has been demonstrated in prenatally stressed adult male rats. We have previously reported that approximately $80 \%$ of prenatally stressed male rats do not exhibit copulation and that no significant changes in nucleus accumbens dopamine release are seen during exposure to estrous females. In the present study, we investigated whether prenatal stress affects sexual motivation in these animals as adults. Pregnant Wistar rats were subjected to immobilization stress for two hours daily from day 15-19 of gestation. The prenatally stressed male offspring at the age of 3 months were allowed contact with receptive female rats for a $30 \mathrm{~min}$ period per week for 10 weeks; then, between the age of 5 and 6 months, their sexual motivation and copulatory activity were measured. Sexual motivation was measured in terms of sexual partner preference. The number of visits and the duration of each visit to an estrous female (stimulus female) or to a sexually active male rat (stimulus male) were recorded. Compared with control males, prenatally stressed male rats showed a significantly lower number of visits and a shorter duration of each visit to stimulus females. Prenatally stressed males showed no preference for male or female stimulus rats in terms of the number of visits and the duration of each visit, whereas control rats showed a significantly higher number of visits and duration of visits to female stimulus rats than male stimulus rats. A significant decrease in copulatory activity was observed in the prenatally stressed male offspring compared with control male rats, with most of the prenatally stressed males failing to show copulation. In vivo microdialysis experiments were performed on the nucleus accumbens with concurrent observation of sexual behavior. The prenatally stressed rats that did not exhibit copulation showed no significant changes in nucleus accumbens dopamine release during exposure to a stimulus male behind a wire-mesh barrier and the amount of dopamine release remained at the basal levels during actual physical
\end{abstract}

${ }^{1}$ These authors contributed equally to this work.

*Corresponding author. Tel: +886-2-23123456x8240; fax: +886-223225007.

E-mail address: yftsai@ha.mc.ntu.edu.tw (Y.-F. Tsai).

Abbreviations: control-C, copulator of control group; control-NC, noncopulator of control group; DA, dopamine; DOPAC, 3,4-dihydroxyphenylacetic acid; $E F$, ejaculation frequency; $E L$, ejaculation latency; HVA, homovanillic acid; IF, intromission frequency; IL, intromission latency; MF, mount frequency; ML, mount latency; NAc, nucleus accumbens; PS, prenatally stressed; PS-C, copulator of prenatally stressed group; PS-NC, non-copulator of prenatally stressed group.

$0306-4522 / 06 \$ 30.00+0.00$ @ 2005 Published by Elsevier Ltd on behalf of IBRO doi:10.1016/j.neuroscience.2005.11.026 contact. These results, combined with those of our previous report, indicate that sexual motivation in prenatally stressed male rats is demasculinized, but not feminized. () 2005 Published by Elsevier Ltd on behalf of IBRO.

Key words: sexual partner preference, microdialysis, nucleus accumbens, dopamine, copulation.

Sexual behavior consists of sexual motivation and copulatory activity, which are regulated by partially overlapping brain areas. Sexual motivation has been considered difficult both to define and measure, despite many attempts over several decades to reveal the underlying mechanisms using a variety of techniques (Beach, 1956; Drewett, 1973; Edwards and Pfeifle, 1983; Everitt et al., 1987; Everitt 1990; Hetta and Meyerson, 1978; Hull et al., 1995; Mendelson and Pfaus, 1989; Meyerson and Lindström, 1973; Paredes and Baum, 1995; Warner et al., 1991).

Exposure of late pregnant rats to immobilization stress has been reported not only to affect male copulatory behavior in most male offspring as adults, but also to increase the frequency of a female lordotic response (Götz and Dörner, 1980; Ward, 1972). Thus, based on the presence of male copulatory performance, prenatally stressed (PS) male rats can be subdivided into copulators and non-copulators (Wang et al., 1995). The underlying mechanism involved in the demasculinized and feminized copulatory behavior caused by prenatal stress is not completely clear (Chapman and Stern, 1978; Kashon et al., 1992; Ward et al., 1986; Gerardin et al., 2005). However, there is evidence that the decreased male copulatory behavior seen in PS male rats is not due to impaired locomotor activity (Masterpasqua et al., 1976) or to the influence of abnormal maternal behavior of their stressed mothers (Herrenkohl and Whitey, 1976).

Numerous studies have supported the view that sexual motivation and copulatory activity in male rats are associated with dopamine (DA) release in the nucleus accumbens (NAc) (Damsma et al., 1992; Fumero et al., 1994; Mas et al., 1990; Pfaus et al., 1990; Pleim et al., 1990; Wenkstern et al., 1993). In a previous study (Wang et al., 1995), we showed that more than $80 \%$ of PS male rats are non-copulators and that no significant change is seen in NAc DA release in these rats during exposure to estrous females, suggesting demasculinization of both copulatory activity and sexual motivation in most PS males. Although no typical proceptive sexual behavioral patterns of estrous female rats, such as soliciting behavior, darting, or ear wiggling, are seen in PS males, they do not resist being mounted and display a high-quality lordotic response (Ward, 1972). In the present study, we used a sexual partner preference test and in vivo 
microdialysis method to investigate whether prenatal stress causes feminization of sexual motivation in PS male rats.

\section{EXPERIMENTAL PROCEDURES}

\begin{abstract}
Animals
All experimental procedures in the present study conformed to the National Institutes of Health Guide for the Care and Use of Laboratory Animals. All efforts were made to minimize animal suffering and reduce the number of animals used. Adult female Wistar rats were housed individually at $22 \pm 1^{\circ} \mathrm{C}$ on a reversed $14: 10$-h light/dark cycle (lights on at 20:00 h) with food and water available ad libitum. From day 15 to day 19 of gestation, the pregnant animals of the PS group $(n=23)$ were immobilized in a $5 \times 5 \times 11 \mathrm{~cm}$ metal cage for $2 \mathrm{~h}$ per day, while the females of the control group $(n=12)$ were left undisturbed for the whole pregnancy period, except for routine maintenance. After weaning, the male pups were kept in groups of three to four and, from 3 months of age, were allowed contact with receptive females for a 30 min period per week for 10 weeks. Based on the display of sexual behavior and the treatment of the pregnant females, the male offspring were subdivided into four groups: (a) control copulators (control-C, $n=24)$, (b) control non-copulators (control-NC, $n=14$ ), (c) prenatally stressed copulators (PS-C, $n=5$ ), and (d) prenatally stressed non-copulators (PS-NC, $n=24$ )
\end{abstract}

\section{Sexual motivation testing}

The tests were carried out between 12:00 and 18:00 h, the observation period for each male rat being $15 \mathrm{~min}$. The testing apparatus consisted of an open-field arena $(80 \times 80 \mathrm{~cm})$ with two boxes $(25 \times 15 \mathrm{~cm})$ on opposite sides, outside of the arena, in which the stimulus animals, a sexually active male or an estrous female, ovariectomized and s.c. implanted with an estradiol benzoate-filled Silastic capsule $(1.98 \mathrm{~mm}$ inner diameter, $3.18 \mathrm{~mm}$ outer diameter), were placed. The partition between the stimulus and the experimental animals consisted of a metal net, allowing the rats to see and smell each other. The number of visits and duration of each visit to each stimulus animals were recorded. Placing of the forelimbs of the experimental animal within an area $(15 \times 25 \mathrm{~cm})$ in front of the stimulus compartments was counted as one visit and the time between the placing of the forelimbs in the area and leaving the area completely taken as the duration of each visit. Each experimental animal was placed in the arena, allowed to adapt $5 \mathrm{~min}$ in the presence of the stimuli, and then tested for $15 \mathrm{~min}$. The arena was cleaned before another animal was introduced. The position of the stimulus animals was changed randomly. The following measurements were recorded: (a) the number of visits to each of the stimulus animals and (b) the total time spent near each of the stimulus animals. The percentage of total time spent and total number of visits to each of the stimulus animals were calculated. Data for each rat, collected from three tests at intervals of one week, were used for statistical analysis.

\section{Copulatory behavior testing}

Copulatory activity was measured in each rat immediately after the sexual motivation test. In the copulatory behavior test, each subject was placed in a $45 \times 35 \times 35 \mathrm{~cm}$ Plexiglas testing chamber and allowed a $5 \mathrm{~min}$ habituation period; then two sexually receptive young female rats (3-5 months), which had been ovariectomized and s.c. implanted with a $5 \mathrm{~mm}$ estradiol benzoate-filled Silastic capsule, were placed in the chamber. Both females were replaced by others every $15 \mathrm{~min}$ to prevent the male from becoming bored by exposure to the same female partners. For a single copulatory test, the mount latency (ML) and intromission latency (IL) were computed as the time from the beginning of the test until the first response, while the ejaculation latency (EL) was calculated as the time from the initial intromission to ejaculation. When these behavioral items did not occur, they were assigned a latency of $30 \mathrm{~min}$ for statistical analyses. The number of mounts, intromissions, and ejaculations in a 30 min observation period were used to calculate the mount frequency (MF), intromission frequency (IF), and ejaculation frequency (EF), respectively. The mean value of three behavioral tests at intervals of one week was calculated for each rat. The test area in the animal room was dimly illuminated by a $60-\mathrm{W}$ red bulb lamp.

\section{Microdialysis and catecholamine analysis}

All male rats were anesthetized with ketamine $(45 \mathrm{mg} / \mathrm{kg}$, i.p.) and sodium pentobarbital $(10 \mathrm{mg} / \mathrm{kg}$, i.p.). A homemade microdialysis probe was mainly constructed as described by Wang et al. (1995). A silica glass tube (external diameter $144 \mu \mathrm{m}$; Future Instruments Trading, Japan) was inserted into the stainless steel tubing (external diameter $650 \mu \mathrm{m}$ ) and secured at the inlet, using epoxy glue. A 2-mm length of polyacrylonitrile dialysis membrane (AN 69 with internal diameter $220 \mu \mathrm{m}$, external diameter $310 \mu \mathrm{m}$, molecular weight cutoff 40,000 , Hospal Medical, France) was sealed at one end and secured between the end of the stainless steel tubing and the silica glass, using epoxy glue. The probe was stereotaxically implanted into the NAc (coordinates of probe tip relative to bregma: AP: $+1.8 \mathrm{~mm}$, DV: $-8.2 \mathrm{~mm}, \mathrm{ML}$ : $+1.4 \mathrm{~mm}$ ) (Paxinos and Watson, 1986), and then secured with dental cement. Recovery rates (mean \pm SEM) were $12.13 \pm 0.62 \%$ for DA, $11.67 \pm 0.55 \%$ for 3,4-dihydroxyphenylacetic acid (DOPAC) and $11.14 \pm 0.69 \%$ for homovanillic acid (HVA). During surgery, the microdialysis probe was perfused continuously with artificial cerebrospinal fluid $(140 \mathrm{mM} \mathrm{NaCl}, 3.0 \mathrm{mM} \mathrm{KCl}, 1.0 \mathrm{mM}$ $\mathrm{MgCl}_{2}, 1.2 \mathrm{mM} \mathrm{CaCl}_{2}$ and $0.04 \mathrm{mM}$ ascorbic acid) at a rate of 1 $\mu \mathrm{L} / \mathrm{min}$. Following surgery, the perfusion rate was decreased to 0.3 $\mu \mathrm{L} / \mathrm{min}$ and the experimental rats individually housed in transparent Plexiglas bowl-cages.

The sexual behavior test, with concurrent microdialysis sampling, was performed one day after surgery during the rats' dark phase. Ninety minutes before dialysate sampling, the perfusion rate was returned to $1 \mu \mathrm{L} / \mathrm{min}$ and microdialysis samples collected every 20 min throughout the experiment. Twenty minutes prior to sampling, the experimental rat was transferred to a transparent Perspex mating chamber $(45 \times 35 \times 35 \mathrm{~cm})$, containing a vertical wire-mesh barrier $(30 \times 30 \mathrm{~cm})$ that divided the chamber into two compartments (compartment A: $30 \times 35 \times 35 \mathrm{~cm}$ and compartment B: $15 \times 35 \times 35 \mathrm{~cm})$. The behavioral test consisted of four consecutive phases as follows: (a) phase I (60 $\mathrm{min})$ : the experimental rat was kept in compartment $A$; (b) phase II (40 min): a sexually active male rat as stimulus animal was placed behind the barrier in compartment B; (c) phase III (40 min): the wire-mesh barrier was removed to allow physically sexual interaction; and (d) phase IV (80 $\mathrm{min})$ : the stimulus male was removed. Microdialysis samples were analyzed by high performance liquid chromatography with electrochemical detection and a mobile phase delivered at a flow rate of $50 \mu \mathrm{L} / \mathrm{min}$ onto a microbore column. The mobile phase consisted of $1.75 \mathrm{~g}$ of heptanesulfonic acid, $0.1 \mathrm{~g}$ of disodium EDTA, $3.5 \mathrm{~mL}$ of triethylamine, $4 \mathrm{~mL}$ of phosphoric acid, and $40 \mathrm{~mL}$ of acetonitrile made up to $1 \mathrm{~L}$ in distilled water and was filtered and degassed just prior to use. The concentration of DA, DOPAC and HVA in dialysate was compared with an external standard.

\section{Histology}

Histological sectioning of the brain with a cryostat confirmed the accuracy of probe placement and only data from those experiments in which the probe was located in the NAc were analyzed.

\section{Statistical analyses}

The chi-square test was used to compare the copulator/noncopulator ratio for the control and PS rats. Comparisons of male copulatory behavioral components between groups were per- 
Table 1. Number of surviving male and female pups of control female rats and female rats exposed to immobilization stress during late pregnancy

\begin{tabular}{lllll}
\hline Group & $n$ & Litter size & Male & Female \\
\hline Control & 12 & $8.1 \pm 0.9^{\mathrm{a}}$ & $3.8 \pm 0.7$ & $4.3 \pm 0.6$ \\
Stress & 10 & $8.2 \pm 0.9$ & $3.7 \pm 0.7$ & $4.5 \pm 0.6$ \\
\hline
\end{tabular}

a Mean \pm SEM.

formed using the Mann-Whitney $U$ test, while Student's $t$-test and paired $t$-test were used to compare sexual motivation. Two-factor repeated measure ANOVA was used to analyze the microdialysis data. A difference with $P<0.05$ was considered significant in all comparisons.

\section{RESULTS}

\section{Effects of immobilization stress on litter size}

Twenty-three pregnant female rats subjected to immobilization stress (experimental group) and 12 controls were used. Three rats from the experimental group died during immobilization stress and another 10 killed, or refused to
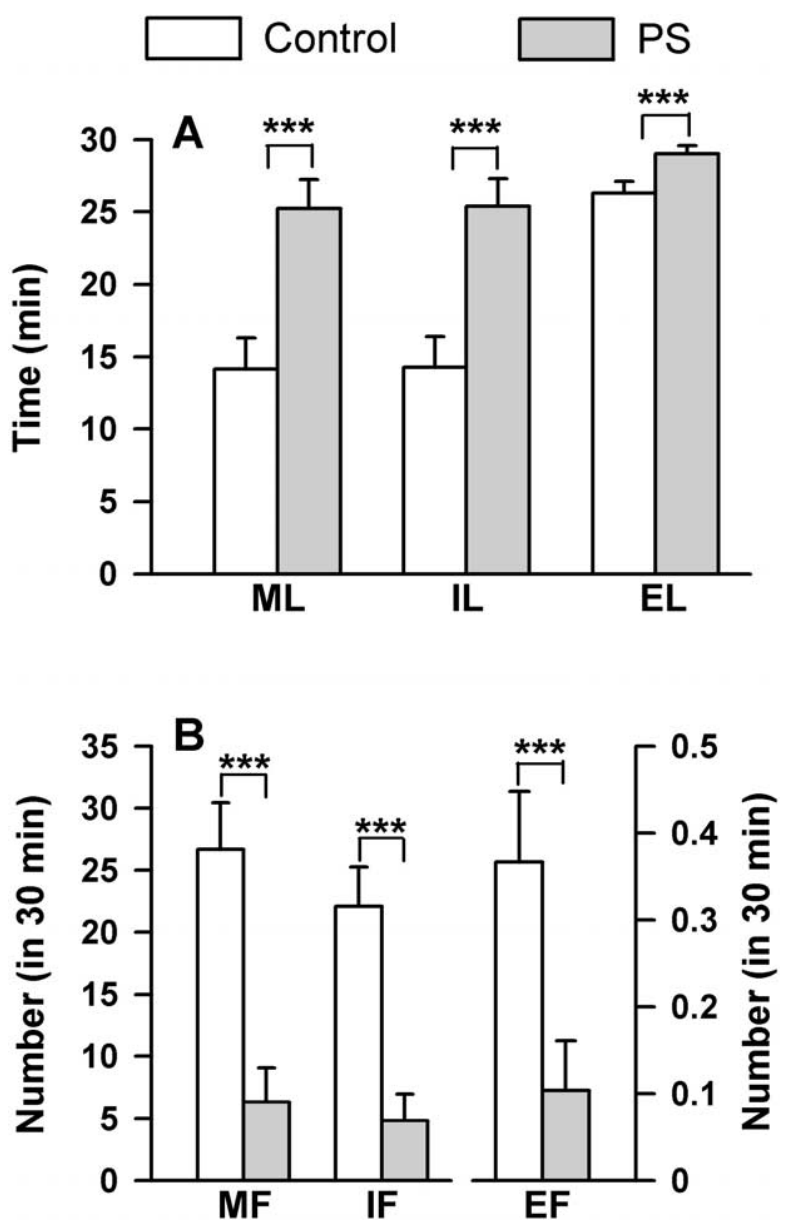

Fig. 1. Effects of prenatal stress on copulatory behavior in all male rats. (A) Mean ( \pm S.E.M.) ML, IL, and EL. (B) Mean ( \pm S.E.M.) MF, IF, and $E F$ in a 30 min behavioral test. Control group $n=38$, PS group $n=29,{ }^{* * *} P<0.001$.
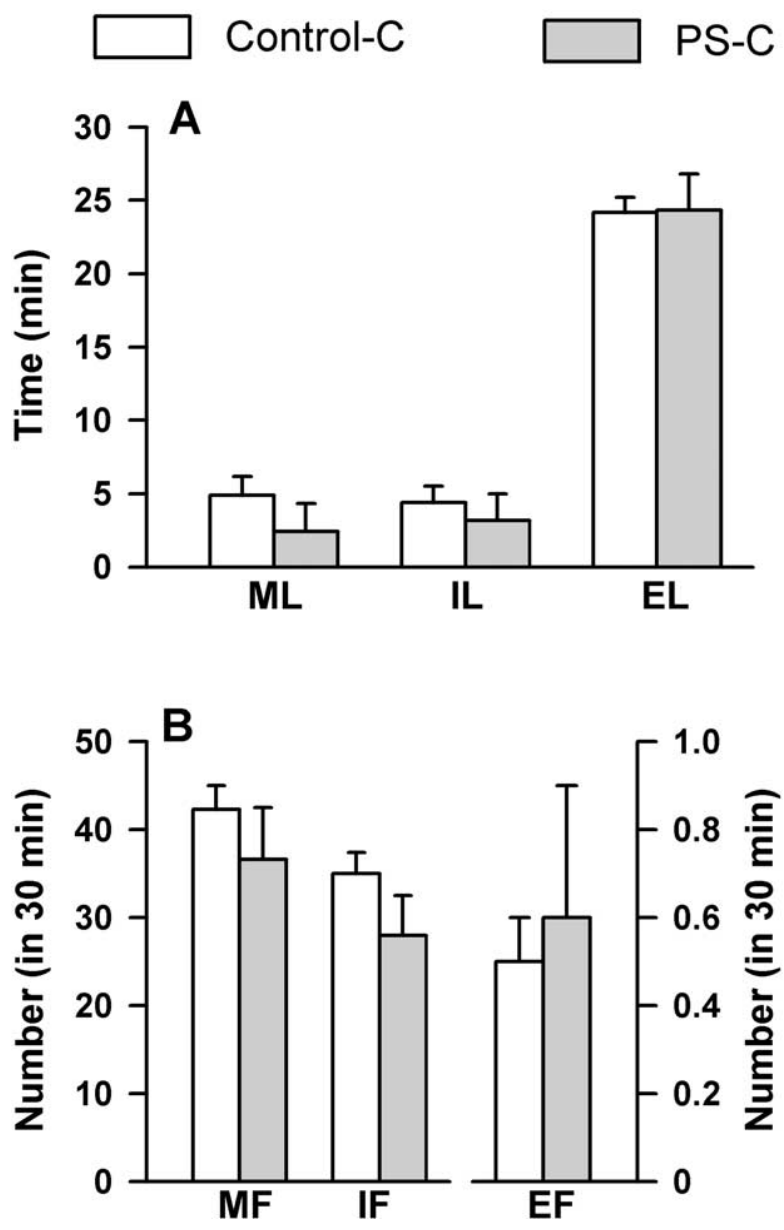

Fig. 2. Effects of prenatal stress on copulatory behavior in copulator male rats. (A) Mean ( \pm S.E.M.) ML, IL, and EL. (B) Mean ( \pm S.E.M.) $\mathrm{MF}, \mathrm{IF}$, and $\mathrm{EF}$ in a $30 \mathrm{~min}$ behavioral test. Control-C group $n=24$, PS-C group $n=5$.

suckle, their pups. The remaining 10 female rats in the experimental group showed maternal behavior and suckled their pups for 21 days. In contrast, all females in the control group suckled their pups. Table 1 shows that there were no differences in pup sex or litter number between the experimental and control groups.

\section{Effects of prenatal stress on male copulatory behavior}

PS rats demonstrated a significant decrease in copulation compared with the control group. As shown in Fig. 1, control rats showed a shorter ML, IL, and EL and a higher MF, IF, and EF than PS rats $(P<0.001)$. More than $80 \%$ of PS rats and approximately $40 \%$ of control rats were noncopulators. The chi-square test showed that exposure of pregnant females to prenatal stress clearly resulted in more non-copulator offspring in adulthood $\left(\chi^{2}=14.12, P<0.01\right)$.

In order to determine whether there were differences in copulatory behavior between PS-C rats and control-C rats, all six behavioral items of copulation were analyzed in these two groups. Fig. 2 shows that the copulatory behavior of the PS-C rats did not differ from that of control-C rats. 

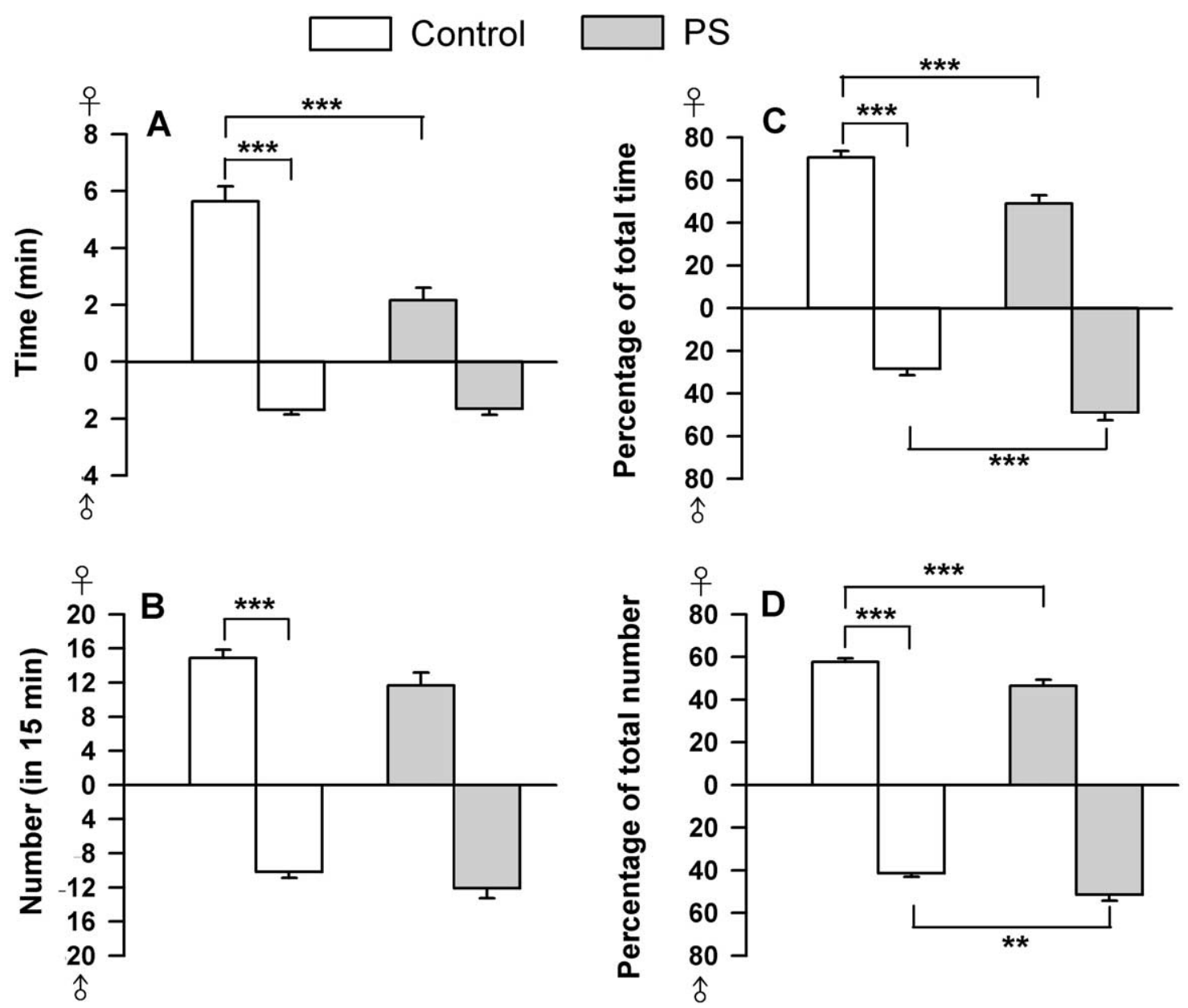

Fig. 3. Effects of prenatal stress on sexual motivation in all male rats. (A) Mean ( \pm S.E.M.) time spent in the vicinity of an active male or estrous female. (B) Mean ( \pm S.E.M.) number of visits paid to the stimulus rats. (C) Mean ( \pm S.E.M.) percentage of the total time spent in the vicinity of an active male or estrous female. (D) Mean ( \pm S.E.M.) percentage of the total number of visits paid to the stimulus rats. Control group $n=38$, PS group $n=29$, ${ }^{* *} P<0.01,{ }^{* * *} P<0.001$.

\section{Effects of prenatal stress on sexual motivation}

As shown in Fig. 3, PS rats did not show any sexual preference in terms of the total time of visits, total number of visits, percentage of the total visit time, or percentage of the total visit numbers, while the control group showed a markedly longer duration and greater frequency of visits to estrous female rats than to sexually active males $(P<0.001)$. Furthermore, based on the percentage of the total time and the percentage of the total number of visits, PS rats spent significantly longer visiting $(P<0.001)$ and had a higher frequency of visits $(P<0.01)$ to sexually active males than did control rats (Fig. $3 C$ and $3 D$ ), i.e. compared with control rats, the PS group showed a significant higher preference for sexually active male rats and a lower preference for estrous females.

\section{Analysis of sexual motivation of PS-NC rats}

Both PS-NC and control-NC rats showed no significant preference for female or male stimulus rats in the sexual motivation test. A similar result was obtained for the per- centage of the total time and the percentage of the total number of visits (Fig. 4).

\section{Analysis of sexual motivation of PS-C rats}

As shown in Fig. 5, control-C rats showed a marked preference for estrous females than for sexually active males $(P<0.001)$, while a statistically significant difference in sexual preference for female versus male rats was seen in PS-C rats only in terms of the percentage of the total time $(P<0.05)$ (Fig. 5C), although they seemed to show a tendency to a greater preference for estrous female rats than sexually active male rats. Compared with control-C rats, PS-C rats spent more total time (Fig. 5A) and number of visits (Fig. $5 B$ ) with the stimulus males. A statistically significant difference in approaching sexually active males was also seen between control-C and PS-C rats in terms of percentage of the total time (Fig. $5 \mathrm{C})(P<0.05)$, but not in terms of the percentage of the total number of visits (Fig. $5 D)$. In addition, there was a statistically significant difference in nearing estrous females between control-C and PS-C rats (Fig. 5C) $(P<0.05)$. 


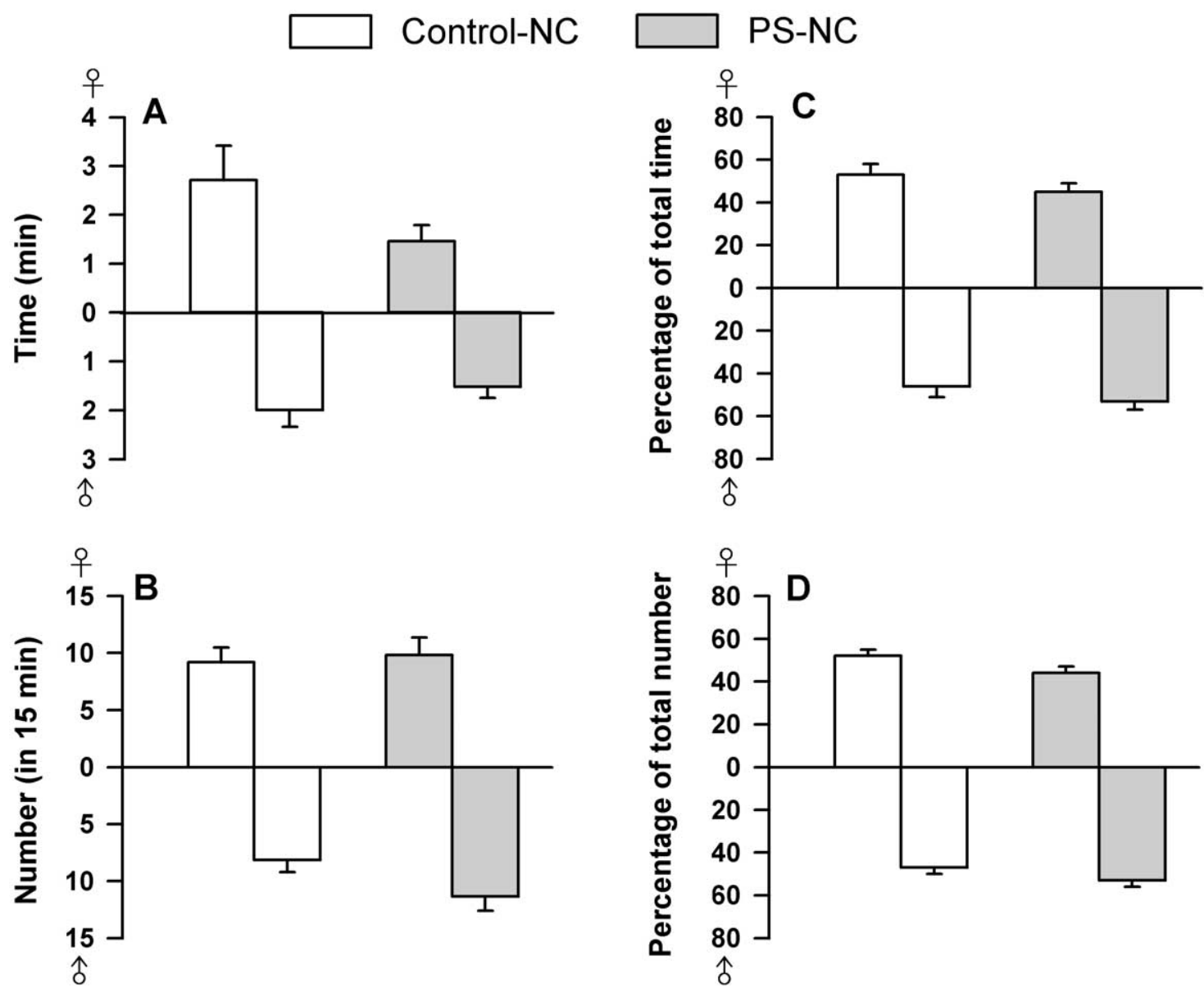

Fig. 4. Effects of prenatal stress on sexual motivation in noncopulator male rats. (A) Mean ( \pm S.E.M.) time spent in the vicinity of an active male or estrous female. (B) Mean ( \pm S.E.M.) number of visits paid to the stimulus rats. (C) Mean ( \pm S.E.M.) percentage of the total time spent in the vicinity of an active male or estrous female. (D) Mean ( \pm S.E.M.) percentage of the total number of visits paid to the stimulus rats. Control-NC group $n=14$, PS-NC group $n=24$.

\section{DA release in the NAc of PS-NC rats after exposure to sexually active male rats}

As shown in Fig. 6, there was no significant difference in extracellular DA, DOPAC, and HVA levels in the NAC between the control-C and PS-NC groups during any of the four phases. During phase III, neither control-C nor PS-NC rats displayed any copulatory activity with sexually active male rats after removal of the wire-mesh barrier.

\section{DISCUSSION}

The significant decrease in copulatory behavior seen in male rats caused by prenatal stress was mainly due to most PS rats' failure to copulate (Fig. 1). PS rats that did display copulation showed no difference in copulatory activity compared with the control-C group (Fig. 2). Since more than $80 \%$ of PS rats were non-copulators, prenatal stress is suggested to cause demasculinization of sexual behavior in male rats. Our present results agree with those in a previous report (Ward, 1972). The reason why some PS rats show complete male copulatory behavior is unclear.
Although the effects of prenatal stress on copulatory behavior in male rats have been extensively studied (Gerardin et al., 2005; Götz and Dörner, 1980; VelazquezMoctezuma et al., 1993; Ward, 1972; Wang et al., 1995), little attention has been paid to the aspect of sexual motivation. The present findings demonstrate that subjection of pregnant female rats to immobilization stress caused significant impairment, not only of male copulatory activity, but also male sexual motivation, in the male offspring during adulthood. Most control male rats showed an obvious preference for estrous female rats, while most PS male rats showed no preference for estrous females or sexually active males. Based on the data for the total time, percentage of the total time, and percentage of the total number of visits, control rats demonstrated a significantly greater female orientation than PS rats (Fig. 3A, 3C and 3D), while PS rats showed a significantly greater maleoriented preference than control rats when sexual preference was measured in terms of the percentage of the total time (Fig. 3C) and percentage of the total number of visits (Fig. 3D). These results indicate that prenatal stress causes demasculinization of sexual motivation in male rats. 


\section{Control-C}
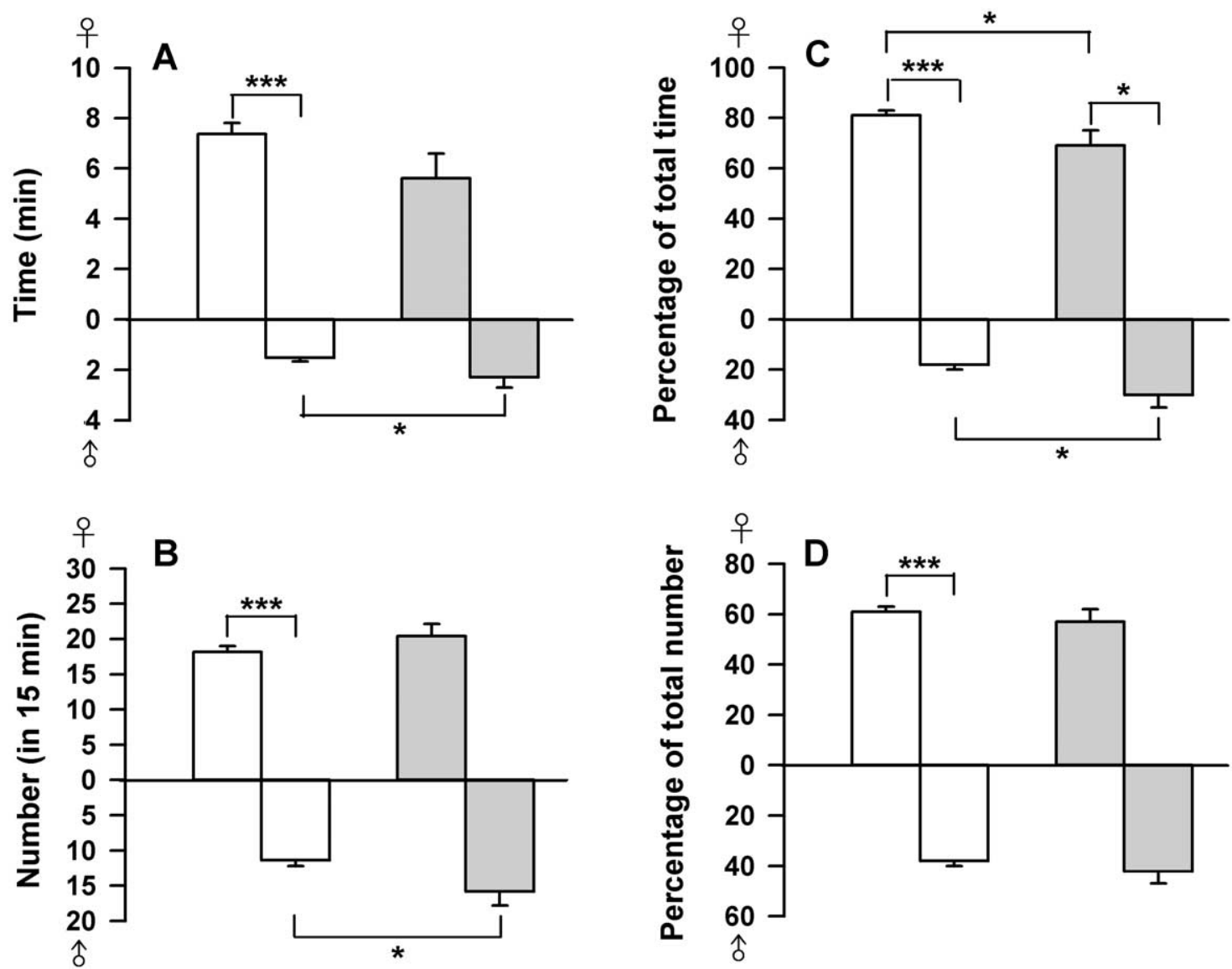

Fig. 5. Effects of prenatal stress on sexual motivation in copulator male rats. (A) Mean ( \pm S.E.M.) time spent in the vicinity of an active male or estrous female. (B) Mean ( \pm S.E.M.) number of visits paid to the stimulus rats. (C) Mean ( \pm S.E.M.) percentage of the total time spent in the vicinity of an active male or estrous female. (D) Mean ( \pm S.E.M.) percentage of the total number of visits paid to the stimulus rats. Control-C group $n=24, \mathrm{PS}-\mathrm{C}$ group $n=5$. *** $P<0.001$ and ${ }^{* *} P<0.01$.

Since both control and PS male rats could be divided into two subgroups according to the presence of copulatory behavior, a further comparison of the sexual motivation between the control and PS group was made. In the non-copulator subgroups, there was no difference in sexual motivation between the control-NC and PS-NC groups, and neither demonstrated any obvious preference for either stimulus males or stimulus females (Fig. 4). These results suggest that the demasculinized copulatory behavior caused by prenatal stress is associated with a lack of female-oriented preference in male rats. In the copulatory subgroups, although no difference in copulatory behavior was seen between the control-C and PS-C groups, PS-C rats spent more total time and visits with sexually active males than control-C rats did, and control- $\mathrm{C}$ rats demonstrated a greater preference than PS-C rats for estrous females (Fig. 5). The PS-C rats showed no statistically significant preference for males or females, but this may be due to the small number of subjects $(n=5)$, as they seemed to show a tendency to prefer females. These findings indicate that prenatal stress may even demasculinize sexual motivation in male rats that display complete copulatory behavior. Velazquez-Moctezuma et al. (1993) reported that prenatal immobilization stress produces demasculinized male rats and feminized copulatory behavior. Our findings indicate that prenatal stress resulted in demasculinization of sexual motivation in male rats, but not in feminization of sexual motivation.

Sexually naïve male rats show no preference for either estrous females or sexually active males, while sexually experienced males show a significant preference for estrous females (Vega-Matuszczyk and Larsson, 1994). In the present study, both the control and PS males were exposed 10 times to estrous females to gain sexual experience before copulatory behavior was recorded. The PS-C rats showed a significant preference for estrous females compared with the PS-NC rats (Fig. 4 and Fig. 5), but the female-oriented preference was still low in PS-C rats compared with control-C rats. In contrast, PS-C rats showed a markedly greater male-oriented preference than control-C rats. Thus, although sexual motivation can be influenced by previous sexual experience in non-PS rats (VegaMatuszczyk and Larsson, 1994), demasculinization of sexual motivation in PS rats is not related to whether they 

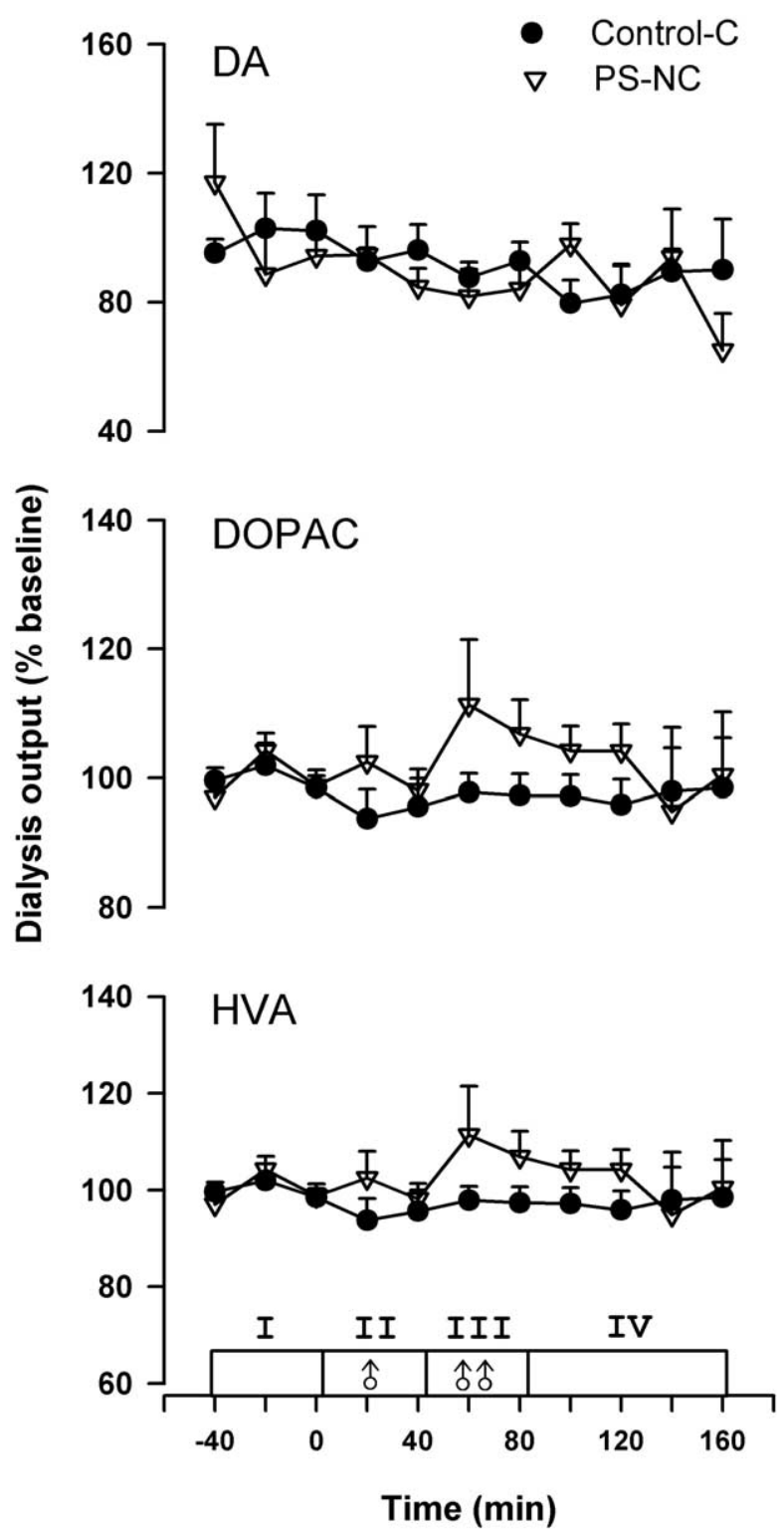

Fig. 6. Temporal changes in dialysate concentrations of DA, DOPAC, and HVA in the NAC of PS-NC and control-C rats during the sexual behavior test. Repeated measures ANOVAs were performed on the percentage change in DA, DOPAC, and HVA in six dialysis samples. The roman numerals indicate the four consecutive phases of the behavioral test. The arrow in phase II indicates the stimulus rat was placed behind the screen and the double arrow in phase III indicates that physical contact was allowed by removal of the screen. Control-C group $n=4$, PS-NC group $n=4$.

have any actual copulatory experience, as both PS-C rats (with sexual experience) and PS-NC rats (without sexual experience) showed an increased preference for stimulus males compared with controls.

In the present study, as shown in Fig. 6, the release of DA and its metabolites, DOPAC and HVA, in the NAC remained at basal levels in control-C males when a sexually active male was placed behind a wire-mesh barrier. In contrast, our previous study showed a significant increase in NAc DA release in control-C males on presentation of an estrous female rat (Wang et al., 1995), while Fumero et al. (1994) found that there was no increase in NAc DA activity in intact male rats exposed to non-estrous female rats. It is therefore postulated that increased DA release in the NAc is associated with sexual motivation in male rats. No significant increase in NAc DA release was seen in either PS-NC or control-C males when a sexually active male rat was placed behind the barrier, indicating that the sexually active male rat fails to induce sexual arousal in either the PS-NC or control-C males. Since female sexual motivation should normally be elicited by males, a sexually active male rat was an appropriate stimulus to examine it and the response of the control-C rats should be a suitable reference.

Although it has been suggested that androgens are involved in female sexual motivation (De Jonge et al., 1986), Williams et al. (1991) reported that female sexual motivation is a distinctly estrogen-mediated response which requires a higher level of estrogen stimulation than sexual receptivity and proceptivity. Prenatal stress does not influence the weight of sexual organs in adult male rats; however, it results in reduced testosterone levels in these animals (Gerardin et al., 2005). These findings suggest that a lack of feminized sexual motivation in PS males may be due to decreased sex hormone levels.

The sexual motivation of an animal is regarded as masculinized when the animal shows a significant preference for estrous females, while it is defined as demasculinized when the female-oriented preference is absent. In contrast, sexual motivation is designated as feminized for an animal that shows an obvious male-oriented preference and as defeminized when it shows no sexual preference for males. Thus, if PS-NC rats developed a female-typical sexual motivation, a significant increase in NAc DA release should be seen in PS-NC rats exposed to sexually active male rats. In addition, male-oriented preference in the sexual motivation test should also be significantly higher than female-oriented preference in PS-NC rats. However, the data collected in the present study show the opposite profile. Thus, our results from both behavioral observation and in vivo microdialysis, combined with those of our previous report, indicate that prenatal stress demasculinizes, but does not feminize, sexual motivation in male rats.

Acknowledgments-This work was supported by the National Science Council grant NSC-93-2413-H002-020, ROC.

\section{REFERENCES}

Beach FA (1956) Characteristics of masculine "sex drive." Nebraska Symp Motivation 4:1-32.

Chapman RH, Stern JM (1978) Maternal stress and pituitary-adrenal manipulations during pregnancy in rats: effects on morphology and sexual behavior of male offspring. J Comp Physiol Psychol 92: 1074-1083.

Damsma G, Pfaus JG, Wenkstern D, Phillips AG, Fibiger HC (1992) Sexual behavior increases dopamine transmission in the nucleus accumbens and striatum of male rats: comparison with novelty and locomotion. Behav Neurosci 106:181-191.

De Jonge FH, Kalverdijk EH, van de Poll NE (1986) Androgens are specifically implicated in female rat sexual motivation. The influ- 
ence of methyltrienelone (R1881) on sexual orientation. Pharmacol Biochem Behav 24:285-289.

Drewett RF (1973) Sexual behavior and sexual motivation in the female rat. Nature 242:476-477.

Edwards DA, Pfeifle JK (1983) Hormonal control of receptivity, proceptivity, and sexual motivation. Physiol Behav 30:437-442.

Everitt BJ (1990) Sexual motivation: a neural and behavioural analysis of the mechanisms underlying appetitive and copulatory responses of male rats. Neurosci Biobehav Rev 14:217-232.

Everitt BJ, Fray P, Kostarczyk E, Taylor S, Stacey P (1987) Studies of instrumental behaviour with sexual reinforcement in male rats (Rattus norvegicus): I. Control by brief visual stimuli paired with a receptive female. J Comp Psychol 101:359-406.

Fumero B, Fernandez-Vera JR, Gonzalez-Mora JL, Mas M (1994) Changes in monoamine turnover in forebrain areas associated with masculine sexual behavior: a microdialysis study. Brain Res 662: 233-239.

Gerardin DCC, Pereira OCM, Kempinas WG, Florio JC, Moreira EG, Bernardi MM (2005) Sexual behavior, neuroendocrine, and neurochemical aspects in male rats exposed prenatally to stress. Physiol Behav 84:97-104.

Götz F, Dörner G (1980) Homosexual behavior in prenatally stressed male rats after castration and estrogen treatment in adulthood. Endokrinologie 76:115-117.

Herrenkohl LR, Whitey JB (1976) Effects of prepartal stress on postpartal nursing behavior, litter development and adult sexual behavior. Physiol Behav 17:1019-1021.

Hetta J, Meyerson BJ (1978) Sexual motivation in the male rat. A methodological study of sex-specific orientation and effects of gonadal hormones. Acta Physiol Scand Suppl 453:1-68.

Hull EM, Du J, Lorrain DS, Matuszewich L (1995) Extracellular dopamine in the preoptic area: implications for sexual motivation and hormonal control of copulation. J Neurosci 15:7465-7471.

Kashon ML, Ward OB, Grisham W, Ward IL (1992) Prenatal endorphin can modulate some aspects of sexual differentiation in rats. Behav Neurosci 106:555-562.

Mas M, Gonzalez-Mora JL, Louilot A, Solé C, Guadalupe T (1990) Increased dopamine release in the nucleus accumbens of copulating male rats as evidenced by in vivo voltammetry. Neurosci Lett 110:303-308.

Masterpasqua F, Chapman RH, Lore RK (1976) The effects of prenatal psychological stress on the sexual behavior and reactivity of male rats. Dev Psychobiol 9:403-411.
Mendelson SD, Pfaus JG (1989) Level searching: a new assay of sexual motivation in the male rat. Physiol Behav 45:337-341.

Meyerson BJ, Lindström LH (1973) Sexual motivation in the female rat: a methodological study applied to the investigation of the effect of estradiol benzoate. Acta Physiol Scand Suppl 389:1-80.

Paredes RG, Baum MJ (1995) Altered sexual partner preference in male ferrets given excitotoxic lesions of the preoptic area/anterior hypothalamus. J Neurosci 15:6619-6630.

Paxinos G, Watson C (1986) The rat brain in stereotaxic coordinates, 2nd edition. Orlando: Academic Press.

Pfaus JG, Damsma G, Nomikos GG, Wenkstern DG, Blaha CD, Phillips AG, Fibiger HC (1990) Sexual behavior enhances central dopamine transmission in the male rat. Brain Res 530:345-348.

Pleim ET, Matochik JA, Barfield RJ, Auerbach SB (1990) Correlation of dopamine release in the nucleus accumbens with masculine sexual behavior in rats. Brain Res 524:160-163.

Vega-Matuszczyk JV, Larsson K (1994) Experience modulates the influence of gonadal hormones on sexual orientation of male rats. Physiol Behav 55:527-531.

Velazquez-Moctezuma J, Salazar EM, Rueda MLC (1993) The effect of prenatal stress on adult sexual behavior in rats depends on the nature of the stressor. Physiol Behav 53:443-448.

Wang CT, Huang RL, Tai MY, Tsai YF, Peng MT (1995) Dopamine release in the nucleus accumbens during sexual behavior in prenatally stressed adult male rats. Neurosci Lett 200:29-32.

Ward IL (1972) Prenatal stress feminizes and demasculinizes the behavior of males. Science 175:82-84.

Ward OB, Monaghan EP, Ward IL (1986) Naltrexone blocks the effects of prenatal stress on sexual behavior differentiation in male rats. Pharmacol Biochem Behav 25:573-576.

Warner RK, Thompson JT, Markowski VP, Loucks JA, Bazzett TJ, Eaton RC, Hull EM (1991) Microinjection of the dopamine antagonist cis-flupenthixol into the MPOA impairs copulation, penile reflexes and sexual motivation in male rats. Brain Res 540: 177-182.

Wenkstern D, Pfaus JG, Fibiger HC (1993) Dopamine transmission increases in the nucleus accumbens of male rats during their first exposure to sexually receptive female rats. Brain Res 618:41-46.

Williams GW, Goldman J, McGinnis MY, Possidente B, Lumia AR (1991) Effects of ovarian hormones on sexual receptivity, proceptivity, and motivation in olfactory bulbectomized female rats. Physiol Behav 50:751-755. 